\title{
Developing industries in cooperative interaction: equilibrium and stability in processes with lag
}

\author{
A.I.Kirjanen, O.A.Malafeyev, N.D.Redinskikh* \\ Faculty of Applied Mathematics and Control Processes, Saint-Petersburg State University, Russia.
}

(Received: 18 August 2017; Accepted: 18 October 2017)

\begin{abstract}
A mathematical model of dynamic interaction between mining and processing industries is formalized and studied in the paper. The process of interaction is described by a system of two delay differential equations. The criterion for asymptotic stability of nontrivial equilibrium point is obtained when both industries co-work steadily. The problem is reduced to finding stability criterion for quasi-polynomial of second order. Time intervals between deliveries of raw materials which make it possible to preserve stable interaction between the two industries are found.
\end{abstract}

Keywords Dynamic cooperative interaction, Mining and processing industries, Delay, Differential equations, Coefficient criteria for asymptotic stability

AMS 2010 subject classifications 34D20, 91A99, 91B55, $91 \mathrm{~B} 76$

DOI: $10.19139 /$ soic.v5i4.357

\section{Introduction}

A mathematical model of dynamic interaction between mining and processing industries is formalized and studied in the paper. It is supposed, that the resource is mined by the first industry and then it is transformed into some product by the second industry. Let us denote the amount of the resources mined by $P$ and the number of companies, producing the final product from this resource. Similarly, it is possible to consider $Q$ as the total output in the processing industry.

Let us assume, that the volume of the mined resources is growing with a coefficient $a>0$ due to ongoing mineral exploration and the amount of raw materials for processing industry is unlimited. We assume that the increase in the number of processing companies leads to the reduction of the volume of extracted raw materials with coefficient $b>0$ and, conversely, an increase in the amount of raw materials implies an increase in the number of processing companies with coefficient $d>0$. Although the extraction of raw materials occurs continuously, it is shipped to producers in portions with some positive time lag h. So the amount of raw materials mined depends on its volume obtained earlier at time moment $(t-h)$ when the last supply was made. If this quantity of "old" mined materials is large, the rate of its extraction must decrease with coefficient $e>0$.

In the absence of raw materials the number of processing companies is reduced with the coefficient $c>0$. In the model described by system (1) the number of processing companies at time moment $t$, also depends on the number of already operating companies at time moment $(t-h)$.

So we get that the process of mutual interaction between industries can be described by the differential equation *Correspondence to: N.D.Redinskikh (Email: redinskich@yandex.ru). Faculty of Applied Mathematics and Control Processes, Saint-
Petersburg State University, Universitetskii prospekt 35, Petergof, Saint Petersburg, Russia 198504.

ISSN 2310-5070 (online) ISSN 2311-004X (print)

Copyright (C) 2017 International Academic Press 
system as follows

$$
\begin{gathered}
\frac{d P(t)}{d t}=(a-e P(t-h)-b Q(t)) P(t) \\
\frac{d Q(t)}{d t}=(-c+d P(t)-f Q(t-h)) Q(t) .
\end{gathered}
$$

This system has a nontrivial equilibrium

$$
P^{*}=\frac{a f+b c}{b d+e f} ; \mathrm{Q}^{*}=\frac{a d-c e}{b d+e f},
$$

provided that

$$
a d>c e .
$$

Our aim is to find conditions on the coefficients of system (1) for stabilizing the equilibrium point (2). This would mean that the first industry produces such a quantity of raw materials that they will be processed by the second industry. In this case, it will not be overstocking in the warehouses and there will be sufficient volumes of raw materials.

Necessary and sufficient criterion for stable coexistence of two competitors was obtained in [6]. Graphically the stability areas were described in the form of multidimensional cones in [16]. In this article, our aim is to express conditions for asymptotic stability in the form of inequalities. In this case these conditions help to solve problems of control and stabilization. A delay effect on the stability of the equilibrium point was studied in [5],[2],[3].

A number of mathematical models describing the interaction between agents based on the game theory was considered in $[1,11,12,13,14,15,17,18]$. The results on the business security, the impact of external factors on the growth of the business are given in [10,9]. Mathematical models for delay-dependent linear systems with multiple time delays, for growing tumor, on optimal properties of special nonlinear and semi-infinite problems arising in parametric optimization, criterion for testing hypothesis about impulse response function, filtering problem for functionals of stationary sequences, stability criteria for high even order delay differential equations were considered in $[7,8][20]-[36]$.

\section{Stability criterion for quasi-polynomial}

By changing variables $x=P-P^{*}, \quad \mathrm{y}=Q-Q^{*}$ in the system (1) and writing down a linear approximation system, we get the characteristic quasi-polynomial and the characteristic equation as follows

$$
H(z)=z^{2} e^{2 z}+c_{1} e^{2 z}+c_{2} z e^{z}+c_{3}=0 .
$$

Here $c_{1}=b d h^{2} P^{*} Q^{*}, \quad c_{2}=\left(e P^{*}+f Q^{*}\right) h, c_{3}=e f h^{2} P^{*} Q^{*}$. To get the conditions under which the roots of the quasi-multinomial (4) lie in the left half-plane, we use Pontryagin and Hermite - Biehler criteria [6, 5, 2, 4, 19].

\section{Theorem 1}

The roots of the quasi-polynomial (4) with positive coefficients lie in the left half-plane if one of two following assertions $A$ or $B$ is fulfilled:

Assertion A:

I.1. $0<c_{1}<\pi^{2}$;

I.2. $0<c_{2}<\frac{z(\dot{y})}{\dot{y}}=\frac{2\left(\dot{y}^{2}-c_{1}\right) \sin \dot{y}}{\dot{y}}$, here $\dot{y} \in\left(\sqrt{c_{1}}, \pi\right)$ is a unique root of the equation $\tan y=\frac{\left(c_{1}-y^{2}\right) y}{c_{1}+y^{2}}$; notice, that $\dot{y} \in\left(\frac{\pi}{2} ; \pi\right)$;

I.3.1. If $y_{1} \in\left(0 ; \frac{\pi}{2}\right)$ is a unique root of the equation $2\left(y^{2}-c_{1}\right) \sin y=c_{2} y$ then the following conditions are fulfilled:

I.3.1.1. $c_{1}+c_{3}<y_{1}^{2}$ and I.3.1.2. $c_{1}+c_{2} \frac{\pi}{2}<c_{3}+\frac{\pi^{2}}{4}$.

I.3.2. If $\mathrm{y}_{1}, y_{2} \in\left(\frac{\pi}{2} ; \pi\right), y_{1}<y_{2}$ are the roots of the equation $2\left(y^{2}-c_{1}\right) \sin y=c_{2} y$ then the following conditions are fulfilled: 
I.3.2.1. $y_{1}^{2}<c_{1}+c_{3}<y_{2}^{2}$ and I.3.2.2. $c_{1}+c_{2} \frac{\pi}{2}>c_{3}+\frac{\pi^{2}}{4}$

Assertion B:

II.1. $\pi^{2}<c_{1}<4 \pi^{2}$;

II.2. $0<c_{2}<\frac{z(\dot{y})}{\dot{y}}=\frac{2\left(\dot{y}^{2}-c_{1}\right) \sin \dot{y}}{\dot{y}}$, here $\dot{y} \in\left(\pi ; \sqrt{c_{1}}\right)$ is the unique root of the equation $\tan y=\frac{\left(c_{1}-y^{2}\right) y}{c_{1}+y^{2}}$; notice, that $\dot{y} \in\left(\pi ; \frac{3 \pi}{2}\right)$;

II.3.1. If $y_{1}<y_{2}$ are the roots of the equation $2\left(y^{2}-c_{1}\right) \sin y=c_{2} y$ and $y_{1}, C_{2} \in\left(\pi ; \sqrt{c_{1}}\right)$, then the following conditions are fulfilled:

II.3.1.1. $y_{1}^{2}<c_{1}+c_{3}<y_{2}^{2}$ and II.3.1.2. $c_{1}+c_{2} \frac{\pi}{2}>c_{3}+\frac{\pi^{2}}{4}$.

II.3.2. If $y_{2} \in\left(\frac{3 \pi}{2} ; \sqrt{c_{1}}\right)$ is the unique root of the equation $2\left(y^{2}-c_{1}\right) \sin y=c_{2} y$ then the following conditions are fulfilled:

II.3.2.1. $c_{1}+c_{3}>y_{2}^{2}$ and II.3.2.2. $\frac{9 \pi^{2}}{4}+c_{2} \frac{3 \pi}{2}+c_{3}<c_{1}$.

Proof

Let us substitute $z=i y$ into the quasi-polynomial (4) and write down its real $F(y)$ and imaginary $G(y)$ parts:

$$
\begin{gathered}
F(y)=\left(-y^{2}+c_{1}\right) \cos 2 y-c_{2} y \sin y+c_{3}, \\
G(y)=\left(-2 y^{2} \sin y+2 c_{1} \sin y+c_{2} y\right) \cos y=\left(c_{2} y-z(y)\right) \cos y=0 .
\end{gathered}
$$

Then derivative $G^{\prime}(y)$ may be written as follows

$$
G^{\prime}(y)=\left(c_{2}-z^{\prime}(y)\right) \cos y-\left(c_{2} y-z(y)\right) \sin y .
$$

Here $z(y)=2\left(y^{2}-c_{1}\right) \sin y$.

From Pontryagin and Hermite - Biehler criteria it is known that the roots of quasi-polinomial (4) have negative real parts if the vector of gain-phase cha-racteristic (amplitude-phase characteristic) $w=H(i y)$ monotonically rotates counterclockwise round the origin with positive rate $[6,5,2,4,19]$. It means that the gain-phase characteristic turning around the origin crosses every line passing through the point $(0,0)$ at the positive angle without touching it $[6,5]$. In this case all roots of the functions $F(y)$ and $G(y)$ are real, simple, alternate and the inequality

$$
F(y) G^{\prime}(y)-F^{\prime}(y) G(y)>0
$$

holds for all $y$. For the stability of quasi-polinomial (4) it is sufficient for the inequality (8) to be valid only at the roots of function $G(y)$. In the future, we will consider the inequality

$$
F(y) G^{\prime}(y)>0
$$

at the roots of the function $G(y)$.

Due to the Pontryagin criterion [19] inequalities (8) and (9) are valid iff the rotation angle of gain-phase characteristic $(F(y) ; G(y))$ around the origin asymptotically tends to $\varphi(-2 \pi k+\varepsilon \leq y \leq 2 \pi k+\varepsilon) \approx(4 k s+r) \pi$ as $k$ tends to infinity. Here $s$ is the degree of quasi-polynomial (4) with respect to $e^{z}$ and $r$ is the degree of quasipolynomial (4) with respect to $z$. In our case $s=r=2$ so, polynomials $F(y)$ and $G(y)$ have each 10 roots on the segment $[-2 \pi+\varepsilon ; 2 \pi+\varepsilon]$. As the function $G(y)$ is odd it can not have more than 9 roots over the segment $[-2 \pi ; 2 \pi]$. One root is $y_{0}=0$, the other ones should lie symmetrically with 4 on each side. The tenth root will be discussed later.

Proof of Assertion A. Note that $\frac{\pi}{2}$ and $\frac{3 \pi}{2}$ are the roots of the function $G(y)$. Other two roots $y_{1}<y_{2} \in(0 ; 2 \pi)$ are the solutions of the equation

$$
z(y)=2\left(y^{2}-c_{1}\right) \sin y=c_{2} y .
$$

Since $z^{\prime}(0)=-2 c_{1}<0$, the equation (10) has no roots for small positive y. On the other hand, the function $z(y)$ crosses the $\mathrm{x}$-axis at the points $y=\sqrt{c_{1}}$ and $y=\pi$, so two cases can occur.

Let $0<c_{1}<\pi^{2}$. Then the function $z(y)=2\left(y^{2}-c_{1}\right) \sin y$ is positive over $\left(\sqrt{c_{1}}, \pi\right)$ and equation (10) has 2 solutions on this interval if the coefficient $A_{2}$ is less than the slope of tangent line to the graph of the function 
$z=z(y)$, drawn from the origin. Then the touch point $\dot{y}$ is the solution of the equation $z(y)=z^{\prime}(y) y$ or $\tan y=\frac{\left(c_{1}-y^{2}\right) y}{c_{1}+y^{2}}$. As we consider the solution of this equation on $\left(\sqrt{c_{1}}, \pi\right)$, so $\tan y<0$ and the touch point $\dot{y} \in\left(\frac{\pi}{2} ; \pi\right)$. In this case equation (10) has two roots $y_{1}<y_{2} \in(0 ; \pi)$ if the condition I.2 of the Theorem 1 is satisfied. To satisfy the Hermite - Biehler conditions it is necessary to have alternation of signs both of derivative $G^{\prime}(y)$ and function $F(y)$ in the roots of $G(y)$ and their multiplication should satisfy condition (9).

For the root $y_{0}=0$ we get $G^{\prime}(0)=2 c_{1}+c_{2}>0, F(0)=c_{1}+c_{3}>0$. Let's assume that other roots of the function $G(y)$ are ordered as follows: $y_{1}<\frac{\pi}{2}<y_{2}<\frac{3 \pi}{2}$. Then $c_{2}<z^{\prime}\left(y_{1}\right)$ and the inequality (7) is equivalent to the condition $G^{\prime}\left(y_{1}\right)<0$. For the root $y_{2}$ the inverse inequality is valid. But $\cos y_{2}<0$ so $G^{\prime}\left(y_{2}\right)<0$. Between these two roots a straight line $z=c_{2} y$ lies under the graph of the function $z=z(y)$ so the inequality $c_{2} y<z(y)$ is valid at the point $y=\frac{\pi}{2}$. From (7) we get $G^{\prime}\left(\frac{\pi}{2}\right)>0$. Taking into account the condition I.1, we obtain $G^{\prime}\left(\frac{3 \pi}{2}\right)>0$. So the signs of the derivative $G^{\prime}(y)$ in roots of function $G(y)$ alternate. The function $F(y)$ has the form $F(y)=c_{1}+c_{3}-y_{j}^{2}, \mathrm{j}=1,2$ at the roots $y_{1}$ and $y_{2}$ of the function $G(y)$. If $c_{1}+c_{3}<y_{1}^{2}$ then the both inequalities $F\left(y_{j}\right)<0, \quad j=1,2$ are valid. Inequality $F\left(\frac{\pi}{2}\right)>0$ corresponds to the condition I.3.1.2 of the theorem 1 and then inequality $F\left(\frac{3 \pi}{2}\right)>0$ is valid as well. So the signs of the function $F(y)$ alternate in roots of $G(y)$.

We can get the tenth root of $G(y)$ by shifting the segment $[-2 \pi ; 2 \pi]$ to the right so that the root of the function $G(y) y_{3} \in\left(2 \pi ; 2 \pi+\frac{\pi}{2}\right)$ will be in this segment. As there are no roots of the function $G(y)$ on interval $\left(-2 \pi ;-\frac{3 \pi}{2}\right)$ we don't lose any of the root of the function $G(y)$ on the left side. So we have constructed a segment of $4 \pi$-length, and there are 10 roots of the function $G(y)$ on this segment.

Let the roots of the function $G(y)$ be ordered as follows: $\frac{\pi}{2}<y_{1}<y_{2}<\frac{3 \pi}{2}$. In this case a straight line $z=c_{2} y$ is located over graph of the function $z=z(y)$ on the interval $\left(0 ; y_{1}\right)$, so at point $y=\frac{\pi}{2}$ inequality $c_{2} \frac{\pi}{2}>z\left(\frac{\pi}{2}\right)$ is valid. From (7) we get $G^{\prime}\left(\frac{\pi}{2}\right)<0$. Similarly to the previous case, $c_{2}<z^{\prime}\left(y_{1}\right)$ and taking into account the inequality $\cos y_{1}<0$ from (7) we obtain $G^{\prime}\left(y_{1}\right)>0$. The inverse inequality $c_{2}>z^{\prime}\left(y_{2}\right)$ with $\cos y_{2}<0$ yields $G^{\prime}\left(y_{2}\right)<0$. Inequality $G^{\prime}\left(\frac{3 \pi}{2}\right)>0$ is also valid, and we get the alternation of signs of the derivative $G^{\prime}(y)$ in the roots of the function $G(y)$. From the condition I.3.2.2 of the Theorem 1 we obtain $F\left(\frac{\pi}{2}\right)<0$ and from inequalities $y_{1}^{2}<c_{1}+c_{3}<y_{2}^{2}$ we obtain $F\left(y_{1}\right)>0$ and $F\left(y_{2}\right)<0$. Inequality $F\left(\frac{3 \pi}{2}\right)>0$ is valid as well. So the signs of the function $F(y)$ in roots of $G(y)$ alternate. Further, all $2 \pi$-long segments will include four roots of the function $G(y)$ and there will be a similar alternation of the signs of the derivative $G^{\prime}(y)$ and the signs of the function $F(y)$ in these roots and inequality (9) is valid as well. Assertion A is proved.

Proof of Assertion B. Let $\pi^{2}<c_{1}<4 \pi^{2}$. Then function $z(y)=2\left(y^{2}-c_{1}\right) \sin y$ is positive on $\left(\pi ; \sqrt{c_{1}}\right)$ and equation (10) has 2 solutions on this interval if coefficient $c_{2}$ is less than the slope of tangent to function graph $z=z(y)$, drawn from the origin. It was noted at the proof of the assertion that the touch point $\dot{y}$ is the solution of the equation $\tan y=\frac{\left(c_{1}-y^{2}\right) y}{c_{1}+y^{2}}$. We consider the solution of this equation on $\left(\pi ; \sqrt{c_{1}}\right)$, so the touch point $\dot{y} \in\left(\pi ; \frac{3 \pi}{2}\right)$. Let the roots of $G(y)$ are ordered as follows $\frac{\pi}{2}<y_{1}<y_{2}<\frac{3 \pi}{2}$. We have considered such sequence of roots in the previous part of the proof but now $\pi<y_{1}$. As before we have $c_{2} \frac{\pi}{2}>z\left(\frac{\pi}{2}\right)$ and from (7) we get $G^{\prime}\left(\frac{\pi}{2}\right)<0$. At the point $y_{1}$ we have $c_{2}<z^{\prime}\left(y_{1}\right)$ and $\cos y_{1}<0$ so the inequality (7) is equivalent to the inequality $G^{\prime}\left(y_{1}\right)>0$. At the point $y_{2}$ we have $c_{2}>z^{\prime}\left(y_{2}\right)$ and $\cos y_{2}<0$, so from (7) we obtain $G^{\prime}\left(y_{2}\right)<0$. Inequality $G^{\prime}\left(\frac{3 \pi}{2}\right)>0$ is fulfilled as well. Inequalities $F\left(\frac{\pi}{2}\right)<0, F\left(y_{1}\right)>0, F\left(y_{2}\right)<0, F\left(\frac{3 \pi}{2}\right)>0$ correspond to the inequalities $c_{1}+c_{2} \frac{\pi}{2}<c_{3}+\frac{\pi^{2}}{4}$, $y_{1}^{2}<c_{1}+c_{3}<y_{2}^{2}$, so the signs of the function $F(y)$ in roots of $G(y)$ alternate. If $\frac{\pi}{2}<y_{1}<\frac{3 \pi}{2}<y_{2}$, and $\pi<y_{1}$, then $G^{\prime}\left(\frac{\pi}{2}\right)<0$. The root $y=\frac{3 \pi}{2}$ lies between two roots $y_{1}<y_{2}$ so the straight line $z=c_{2} y$ is located under the graph of the function $z=z(y)$ or $c_{2} y<z(y)$. From this inequality we obtain $G^{\prime}(y)=-\sin y\left(c_{2} y-z(y)<0\right.$ for $y=\frac{3 \pi}{2}$. So the signs of the derivative $G^{\prime}(y)$ in roots of function $G(y)$ alternate. The corresponding alternation of signs of function $F(y)$ takes place iff the inequalities $\frac{9 \pi^{2}}{4}+c_{2} \frac{3 \pi}{2}+c_{3}<c_{1}$ and $c_{1}+c_{3}>y_{2}$ are satisfied. If $\frac{\pi}{2}<\frac{3 \pi}{2}<y_{1}<y_{2}$, then the both inequalities $c_{1}+c_{3}<y_{1}$ and $c_{1}+c_{3}>y_{2}$ are fulfilled together. It contradicts to the inequality $y_{1}<y_{2}$. So the assertion $\mathrm{B}$ of the Theorem 1 is proved.

If $c_{1}>4 \pi^{2}$, the segment $[0 ; 2 \pi]$ contains only two roots of the function $G(y)$, whereas Pontryagin criterion requires four roots. 


\section{Criterion for stable co-functioning of the two industries}

In this paragraph we obtain conditions under which the goods quantity meets the demand. Under these conditions there is as overproducing of raw materials and its shortage as well. Proving the theorem 1 we have considered only linear system associated with the equilibrium point $\left(P^{*} ; Q^{*}\right)$ without square members. From the theory of the differential equations it is known that in this case the equilibrium point of system (1) is asymptotically stable if the approximating linear system is asymptotically stable. The last statement is valid if the roots of the quasi-polynomial (4) lie in the left half-plane. Thus, we obtain the following theorem.

\section{Theorem 2}

A non-trivial equilibrium $\left(P^{*} ; Q^{*}\right)$ of the system (1) is asymptotically stable iff the following conditions are fulfilled:

Assertion A:

I.1. $0<b d h^{2} P^{*} Q^{*}<\pi^{2}$

I.2. $0<\left(e P^{*}+f Q^{*}\right) h<\frac{z(\dot{y})}{\dot{y}}=\frac{2\left(\dot{y}^{2}-b d h^{2} P^{*} Q^{*}\right) \sin \dot{y}}{\dot{y}}$, here $\dot{y} \in\left(\sqrt{b d h^{2} P^{*} Q^{*}}, \pi\right)$ is the unique root of the equation $\tan y=\frac{\left(b d h^{2} P^{*} Q^{*}-y^{2}\right) y}{b d h^{2} P^{*} Q^{*}+y^{2}}$;

I.3.1. If $y_{1} \in\left(0 ; \frac{\pi}{2}\right)$ is a root of equation $2\left(y^{2}-b d h^{2} P^{*} Q^{*}\right) \sin y=\left(e P^{*}+f Q^{*}\right) h y$ then the following conditions are fulfilled:

I.3.1.1. $b d h^{2} P^{*} Q^{*}+e f h^{2} P^{*} Q^{*}<y_{1}^{2}$ and

I.3.1.2. $b d h^{2} P^{*} Q^{*}+\left(e P^{*}+f Q^{*}\right) h \frac{\pi}{2}<e f h^{2} P^{*} Q^{*}+\frac{\pi^{2}}{4}$.

I.3.2. If $y_{1}, y_{2} \in\left(\frac{\pi}{2} ; \pi\right), y_{1}<y_{2}$ are the roots of the equation $2\left(y^{2}-b d h^{2} P^{*} Q^{*}\right) \sin y=\left(e P^{*}+f Q^{*}\right) h y$ then the following conditions are fulfilled:

I.3.2.1. $y_{1}^{2}<b d h^{2} P^{*} Q^{*}+e f h^{2} P^{*} Q^{*}<y_{2}^{2}$ and

I.3.2.2. $b d h^{2} P^{*} Q^{*}+\left(e P^{*}+f Q^{*}\right) h \frac{\pi}{2}>e f h^{2} P^{*} Q^{*}+\frac{\pi^{2}}{4}$

Assertion B:

A non-trivial equilibrium $\left(P^{*} ; Q^{*}\right)$ of the system (1) is asymptotically stable if the following conditions are fulfilled:

II.1. $\pi^{2}<b d h^{2} P^{*} Q^{*}<4 \pi^{2}$;

II.2. $0<\left(e P^{*}+f Q^{*}\right) h<\frac{z(\dot{y})}{\dot{y}}=\frac{2\left(\dot{y}^{2}-b d h^{2} P^{*} Q^{*}\right) \sin \dot{y}}{\dot{y}}$, here $\dot{y} \in\left(\pi ; \sqrt{b d h^{2} P^{*} Q^{*}}\right)$ is the unique root of the equation $\tan y=\frac{\left(b d h^{2} P^{*} Q^{*}-y^{2}\right) y}{b d h^{2} P^{*} Q^{*}+y^{2}}$;

II.3.1. If $y_{1}, y_{2} \in\left(\pi ; \frac{3 \pi}{2}\right) y_{1}<y_{2}$ are the roots of the equation $2\left(y^{2}-b d h^{2} P^{*} Q^{*}\right) \sin y=\left(e P^{*}+f Q^{*}\right) h y$ then the following conditions are valid:

II.3.1.1. $y_{1}^{2}<b d h^{2} P^{*} Q^{*}+e f h^{2} P^{*} Q^{*}<y_{2}^{2}$ and

II.3.1.2. $b d h^{2} P^{*} Q^{*}+\left(e P^{*}+f Q^{*}\right) h \frac{\pi}{2}>e f h^{2} P^{*} Q^{*}+\frac{\pi^{2}}{4}$.

II.3.2. If $y_{2} \in\left(\frac{3 \pi}{2} ; \sqrt{b d h^{2} P^{*} Q^{*}}\right)$ is a root of the equation $2\left(y^{2}-b d h^{2} P^{*} Q^{*}\right) \sin y=\left(e P^{*}+f Q^{*}\right) h y$ then the following conditions are valid:

II.3.2.1. $b d h^{2} P^{*} Q^{*}+e f h^{2} P^{*} Q^{*}>y_{2}^{2}$,

II.3.2.2. $\frac{9 \pi^{2}}{4}+\left(e P^{*}+f Q^{*}\right) h \frac{3 \pi}{2}+e f h^{2} P^{*} Q^{*}<b d h^{2} P^{*} Q^{*}$.

\section{Conclusion and Discussion}

1. A mathematical model of dynamic interaction between mining and processing industries is described by the system of two nonlinear delay differential equations. At the proposed model we take into account the volume of raw materials mined and shipped in the preceding time $(t-h)$. In the Theorem 2 we find conditions on the coefficients of system (1) for stabilizing the equilibrium point (2). It means that the first industry produces such a quantity of raw materials that they will immediately be processed by the second industry. It means that there is a balance between the amount of extracted raw materials and the number of processing enterprises. 
2. The conditions under which the solutions of system (1) tend to equilibrium point $\left(Q^{*} ; P^{*}\right)$ are given in Theorem 2. The conditions I.1, I.2, I.3.1.1 and I.3.1.2 taken together, give a size of admissible interval between deliveries of raw material at which the balance between industries is kept. The size of this $h^{2}<\frac{y_{1}^{2}}{(b d+e f) P^{*} Q^{*}}$, where $y_{1} \in\left(0 ; \frac{\pi}{2}\right)$ is a root of equation $2\left(y^{2}-b d h^{2} P^{*} Q^{*}\right) \sin y=\left(e P^{*}+f Q^{*}\right) h y$.

3. If the interval between deliveries of raw material is not small but conditions I.1, I.2, I.3.2.1 and I.3.2.2 of Theorem 2 taken together are fulfilled then the balance between industries is kept also. In this case size of this admissible interval is $\frac{y_{1}^{2}}{(b d+e f) P^{*} Q^{*}}<h^{2}<\frac{y_{2}^{2}}{(b d+e f) P^{*} Q^{*}}$, where $y_{1}, y_{2} \in\left(\frac{\pi}{2} ; \pi\right), y_{1}<y_{2}$ are the roots of the equation $2\left(y^{2}-b d h^{2} P^{*} Q^{*}\right) \sin y=\left(e P^{*}+f Q^{*}\right) h y$.

4. Moreover if delay $h$ is rather large $\pi^{2}<b d h^{2} P^{*} Q^{*}<4 \pi^{2}$ (condition II.I of Theorem 2) the stability of the equilibrium point $\left(Q^{*} ; P^{*}\right)$ can be restored if conditions of Assertion B of Theorem 2 are fulfilled.

5. If this time interval between supplies of raw materials increases, the asymptotic stability of equilibrium point stabilization. Restrictions obtained in the Theorem 2 allow to solve the problem of stabilization of equilibrium point.

6. Assume that a priori it is known the quantity of final products required to meet demand in the region, and to manifacture these final products we need a certain amount of raw materials. It means that initially the equilibrium point is known, but the point $\left(Q^{*} ; P^{*}\right)$ determined by the formula $(2)$, does not meet the demand for these products. In this case, it is necessary to change the technological process of extraction and processing (change coefficients $a, b, c, d, e, f)$ so that point $\left(Q^{*} ; P^{*}\right)$ is consistent with the economically justified demand. Then it is possible to solve the above mentioned problem of finding time intervals between deliveries of raw materials which make it possible to preserve stable interaction between the two industries.

\section{REFERENCES}

1. X. Grigorieva and O.A. Malafeev, Competitive many-period postman problem with varying parameters, Applied Mathematical Sciences, vol. 8, no. 145-148, pp. 7249-7258, 2014.

2. A. Kirjanen and V. Veselov, The influence of capital turnover time and coefficient criteria of asymptotic stability of two competing firms, Proceedings of V International Conference State and Business, pp. 50-52, 2013.

3. A. Kirjanen and O. Shalyapina, Pointwise synthes controllability of systems with delay, Contemporary Engineering Sciences, vol. 2, pp. 14-18, 1989.

4. J. K. Hale, Theory of functional differential difference equations, Springer Verlag, New York, 1977.

5. A. I. Kirjanen, Stability of systems with aftereffect and there applications, St.Petersburg State University, Russia, Saint-Petersburg, 1994.

6. A.Kirjanen, Coefficient criteria of stable coexistence of two competitors, Stability and Control Processes 2015 - Proceedings, pp.463-466, 2015.

7. L.A. Bondarenko and A.V. Zubov and V.B. Orlov and V.A. Petrova and N.S. Ugegov, Application in practice and optimization of industrial information systems, Journal of Theoretical and Applied Information Technology, vol. 85, no.3, pp. 305-308, 2016.

8. A.V.Zubov and A.Y. Murashko and L.G. Kolyada and E.A. Volkova and O.A. Zubova, Fidelity issue of engineering analysis and computer aided calculations in sign models of dynamic systems, Global Journal of Pure and Applied Mathematics, vol. 12, no.5, pp. 4203-4217, 2016.

9. A.I. Kirjanen and A.A. Samodurov, Influence of external factors on business process of company in dynamics, Proc. of the 7th International Research and Practical Conference, pp. 88-92, 2015.

10. A.I.Kirjanen and A.V.Labudin and A.A.Samodurov, Business-Process Approach to Economic Security Management of the Enterprise with Attraction of the Equation of Profitability, Russian Public Policy Journal (RuPP) Upravlenceskoe konsultirovanie, vol. 3, pp. 96-105, 2016.

11. V.N.Kolokoltsov and O.A.Malafeyev, Mean-Field-Game Model of Corruptions, Dynamic Games and Applications, vol. 7, no.1, pp. 34-47, 2017.

12. O.A. Malafeyev and N.D. Redinskikh and G.V. Alferov, Electric circuits analogies in economics modeling: Corruption networks, BDO 2014 - Proceedings, http://dx.doi.org/10.1109/Emission.2014.6893965, 2014.

13. O.A. Malafeyev and L.A. Petrosyan, Differential search games - Dynamic-games with complete information Vestnik Leningradskogo Universiteta, Seriya Matematika, Mekhanika, Astronomiya, vol. 2, pp. 26-30, 1983.

14. O.A. Malafeev, Existence of equilibrium points in differential noncooperative many-person games, Vestnik Leningradskogo Universiteta, Seriya Matematika, Mekhanika, Astronomiya, vol.3, pp.40-46, 1982.

15. O.A. Malafeev, On the existence of equilibrium points in 2-person differential games with separated dynamics, Vestnik Leningradskogo Universiteta, Seriya Matematika, Mekhanika, Astronomiya, pp.12-16, 1980.

16. V.V. Malygina and M.M. Kipnis, The stability cone for a matrix delay difference equation, International Journal of Mathematics and Mathematical Sciences, http://dx.doi.org/10.1155/2011/860326, 2011.

17. E.G. Neverova and O.A. Malafeyef, A model of interaction between anticorruption authority and corruption groups, AIP Conference Proceedings, http://dx.doi.org/10.1063/1.4912671, 2015 
18. Yu.A. Pichugin and O.A. Malafeev, Statistical estimation of corruption indicators in the firm, Applied Mathematical Sciences, vol.10, no.42, pp.2065-2073, 2016.

19. L.S. Pontryagin, On the zeros of some elementary transcendental functions, American Mathematical Society, vol.2, pp. 95-110, 1955.

20. Y.V.Kozachenko and I.V.Rozora, A criterion for testing hypothesis about impulse response function, Statistics, Optimization and Information Computing, vol. 4, pp. 214-232, 2016.

21. O.Kostyukova and T.Tchemisova and M.Kurdina, On optimal properties of special nonlinear and semi-infinite problems arising in parametric optimization, Statistics, Optimization and Information Computing, vol. 5, pp. 99-108, 2017.

22. N.N. Subbotina and E.A. Kolpakova, Method of Characteristics for Optimal Control Problems and Conservation Laws, Journal of Mathematical Sciences, vol. 199, no.5, pp. 588-595, 2014.

23. M.Luz and M.Moklyachuk, Filtering problem for functionals of stationary sequences, Statistics, Optimization and Information Computing, vol.4, pp.68-83, 2016.

24. O.A.Malafeev and S.A.Nemnyugin, Generalized dynamic model of a system moving in an external field with stochastic components, Theoretical and Mathematical Physics, vol.107, pp.770-774, 1996.

25. E.G.Neverova and O.A.Malafeyev and G.V.Alferov and T.E.Smirnova, Model of interaction between anticorruption authorities and corruption groups, Stability and Control Processes 2015 - Proceedings, pp.488-490, 2015.

26. G.V.Alferov and O.A.Malafeyev, The robot control strategy in a domain with dynamical obstacles, Lecture Notes in Computer Science (including subseries Lecture Notes in Artificial Intelligence and Lecture Notes in Bioinformatics), vol.1093, pp.211-217, 1996.

27. O.A. Malafeev, Stationary strategies in differential games, USSR Computational Mathematics and Mathematical Physics, vol.17, pp.37-46, 1977.

28. O.A.Malafeev, Equilibrium situations in dynamic games, Cybernetics, vol.10, pp.504-513, 1974.

29. Z.Taushanov and A.Berchtold, A direct local search method and its application to a markovian model, Statistics, Optimization and Information Computing, vol. 5, no. 1, pp. 19-34, 2017.

30. Y.V.Kozachenko and M.Y.Petranova, Proper complex random processes, Statistics, Optimization and Information Computing, vol 5, no. 2, pp. 137-146, 2017.

31. M.Moklyachuk and M.Sidei, Filtering problem for stationary sequences with missing observations, Statistics, Optimization and Information Computing, vol. 4, no. 4, pp. 308-325, 2016.

32. X.Naihua, A SQP method for general nonlinear complementarity problems, Applied Mathematics, vol.15, no.4, pp. 433-442, 2000.

33. X.Naihua, A class of trust region methods for linear inequality constrained optimization and its theory analysis: I. Algorithm and global convergence, Applied Mathematics, vol.10, no.3, pp. 287-296, 1995.

34. O.A.Malafeyev and S.A.Nemnyugin and G.A.Ivaniukovich, Stochastic models of social-economic dynamics, Stability and Control Processes 2015 - Proceedings, pp.483-485, 2015.

35. G.D.Drozdov and O.A.Malafeyev and S.A.Nemnyugin, Multicomponent dynamics of competitive single-sector economy development, Stability and Control Processes 2015 - Proceedings, pp.457-459, 2015

36. O.A.Malafeev, The existence of situations of $\varepsilon$-equilibrium in dynamic games with dependent movements, USSR Computational Mathematics and Mathematical Physics, vol.14, pp.88-99, 1974. 